\title{
ISOLATED TOUGHNESS AND PATH-FACTOR UNIFORM GRAPHS
}

\author{
Sizhong Zhou ${ }^{1, *}$, Zhiren Sun ${ }^{2}$ And HongXia LiU ${ }^{3}$
}

\begin{abstract}
A $P_{\geq k}$-factor of a graph $G$ is a spanning subgraph of $G$ whose components are paths of order at least $k$. We say that a graph $G$ is $P_{\geq k}$-factor covered if for every edge $e \in E(G), G$ admits a $P_{\geq k}$-factor that contains $e$; and we say that a graph $G$ is $P_{\geq k}$-factor uniform if for every edge $e \in E(G)$, the graph $G-e$ is $P_{\geq k}$-factor covered. In other words, $G$ is $P_{\geq k}$-factor uniform if for every pair of edges $e_{1}, e_{2} \in E(G), G$ admits a $P_{\geq k}$-factor that contains $e_{1}$ and avoids $e_{2}$. In this article, we testify that (i) a 3-edge-connected graph $G$ is $P_{\geq 2}$-factor uniform if its isolated toughness $I(G)>1$; (ii) a 3-edgeconnected graph $G$ is $P_{\geq 3}$-factor uniform if its isolated toughness $I(G)>2$. Furthermore, we explain that these conditions on isolated toughness and edge-connectivity in our main results are best possible in some sense.
\end{abstract}

Mathematics Subject Classification. 05C70, 05C38, 90B10.

Received December 17, 2019. Accepted April 11, 2021.

\section{INTRODUCTION}

The vulnerability and ruggedness of computer networks are hot topics in network security research and have attracted the wide attention of scholars (see $[1,3]$ ). We simulate the network by a graph, vertices of the graph stands for nodes of the network and edges of the graph acts for links between the nodes of the network. From the perspective of graph theory, for a graph with the same number of vertices, the larger the size of the vertex-cut or edge-cut is, the higher the density and strength of the graph is, and so the stronger the ability to resist attacks in the corresponding network is (see [10,11]). Yang et al. [16] introduced isolated toughness, which is defined in the behind, to measure the stability and vulnerability of networks. Isolated toughness describes a vertex-cut with as few vertices as possible to get as many isolated vertices as possible. In terms of this definition of isolated toughness, we naturally imagine star network denoted by $K_{1, n}$. As long as the central site is attacked, all other sites will be isolated and lose contact with each other. In data transmission networks, the data transmission between two sites go through a path between two corresponding vertices. Hence, the availability of data transmission in the network is equivalent to the existence of path factor of the corresponding graph which is generated by the network. Research on the existence of path factors under specific network structures can help scientists design and construct networks with high data transmission rates. We find that there are strong essential connection between isolated toughness and the existence of path factors in graphs,

Keywords. Graph, isolated toughness, edge-connectivity, path-factor, path-factor uniform graph.

1 School of Science, Jiangsu University of Science and Technology, Zhenjiang, Jiangsu 212100, P.R. China.

2 School of Mathematical Sciences, Nanjing Normal University, Nanjing, Jiangsu 210046, P.R. China.

3 School of Mathematics and Informational Science, Yantai University, Yantai, Shandong 264005, P.R. China.

*Corrresponding author: zsz_cumt@163.com 
and hence investigations on isolated toughness can yield theoretical guidance to meet data transmission and network security requirements.

In this article, a graph is assumed to be undirected, finite and simple. We use $G=(V(G), E(G))$ to denote a graph, where $V(G)$ is its vertex set and $E(G)$ is its edge set. For any $x \in V(G)$, we denote by $d_{G}(x)$ the degree of $x$ in $G$. Let $X$ be a vertex subset of $G$. Denote by $G[X]$ the subgraph of $G$ induced by $X$, and $G-X=G[V(G) \backslash X]$. Let $E^{\prime}$ be an edge subset of $G$. Denote by $G-E^{\prime}$ the subgraph gained from $G$ by deleting $E^{\prime}$. Especially, we write $G-u=G-\{u\}$ for $u \in V(G)$ and $G-e=G-\{e\}$ for $e \in E(G)$. A set $X \subseteq V(G)$ is called independent if no two vertices in $X$ are adjacent to each other, $i(G)$ denotes the number of isolated vertices of $G$, and $\omega(G)$ denotes the number of connected components of $G$. Denote by $P_{n}$ the path of order $n$ and by $K_{n}$ the complete graph of order $n$. Yang et al. [16] defined a graphic parameter, isolated toughness of a graph $G$, denoted by $I(G)$, i.e.,

$$
I(G)=\min \left\{\frac{|X|}{i(G-X)}: X \subseteq V(G), i(G-X) \geq 2\right\}
$$

if $G$ is not a complete graph; otherwise, $I(G)=+\infty$.

A $P_{\geq k}$-factor of a graph $G$ is a spanning subgraph of $G$ whose components are paths of order at least $k$. We say that a graph $G$ is $P_{\geq k}$-factor covered if for every edge $e \in E(G), G$ admits a $P_{\geq k}$-factor that contains $e$.

Akiyama et al. [2] put forward a criterion for a graph having a $P_{\geq 2}$-factor. Wang [13] characterized a bipartite graph with a $P_{\geq 3}$-factor. Kaneko [7] posed a characterization for a graph with a $P_{\geq 3}$-factor. Kano et al. [8] raised a new proof of Kaneko's result. Zhou [19,20], Zhou et al. [24] and Gao et al. [6] gave some sufficient conditions for graphs to have $P_{\geq 3}$-factors with given properties. Kano et al. [9] showed two results for graphs to admit path and cycle factors. Zhang and Zhou [17] derived two necessary and sufficient conditions for graphs being $P_{\geq 2}$-factor covered and $P_{\geq 3}$-factor covered. Zhou [18] showed two binding number conditions for graphs to be $P_{\geq 2}$-factor covered and $P_{\geq 3}$-factor covered. Zhou and Sun [22] obtained two sufficient conditions on the existence of $P_{\geq 2}$-factor and $P_{\geq 3}$-factor uniform graphs. Gao and Wang [4] and Gao et al. [5] established some relationships between isolated toughness and graph factors. Zhou et al. [28] showed two existence theorems for graphs to admit component factors. Zhou et al. [27], Sun and Zhou [12] and Wang and Zhang [15] investigated the existence of edge-disjoint factors in graphs and gained some results for graphs to possess edge-disjoint factors. Other results on graph factors can be found in $[14,21,23,25,26,29]$.

A graph $G$ is called a $P_{\geq k}$-factor uniform graph if for every edge $e \in E(G)$, the graph $G-e$ is $P_{\geq k}$-factor covered. In other words, $G$ is $P_{\geq k}$-factor uniform if for every pair of edges $e_{1}, e_{2} \in E(G), G$ admits a $P_{\geq k}$-factor that contains $e_{1}$ and avoids $e_{2}$. In this article, we gain two isolated toughness conditions for the existence of $P_{\geq 2}$-factor uniform graphs and $P_{\geq 3}$-factor uniform graphs, which are given in Sections 2 and 3 . The proofs of our main results depend on the following two lemmas.

Lemma 1.1 ([17]). A connected graph $G$ is $P_{\geq 2}$-factor covered if and only if

$$
i(G-X) \leq 2|X|-\varepsilon_{1}(X)
$$

for arbitrary $X \subseteq V(G)$, where $\varepsilon_{1}(X)$ is defined as

$$
\varepsilon_{1}(X)= \begin{cases}2, & \text { if } X \text { is not an independent set; } \\ 1, & \text { if } X \text { is a nonempty independent set, and } G-X \text { contains } \\ 0, & \text { a nontrivial component; }\end{cases}
$$

A graph $W$ is factor-critical if $W-x$ contains a perfect matching $\left(\left\{P_{2}\right\}\right.$-factor $)$ for all $x \in V(W)$. A graph $D$ is called a sun if $D=K_{1}, D=K_{2}$ or $D$ is the corona of a factor-critical graph $W$ with order at least 3, that $i s, D$ is obtained from $W$ by adding a new vertex $z=z(y)$ together with a new edge yz for every $y \in V(W)$. $A$ sun of order $n$ with $n \geq 6$ is called a big sun. Denote the number of sun components of $G$ by $\operatorname{sun}(G)$. 
Lemma $1.2([17])$. A connected graph $G$ is $P_{\geq 3}$-factor covered if and only if

$$
\operatorname{sun}(G-X) \leq 2|X|-\varepsilon_{2}(X)
$$

for arbitrary subset $X$ of $V(G)$, where $\varepsilon_{2}(X)$ is defined as

$$
\varepsilon_{2}(X)= \begin{cases}2, & \text { if } X \text { is not an independent set; } \\ 1, & \text { if } X \text { is a nonempty independent set, and } G-X \text { contains } \\ 0, & \text { a non-sun component; }\end{cases}
$$

\section{Isolated TOUGHNESS AND $P_{\geq 2}$-FACTOR UNIFORM GRAPHS}

Theorem 2.1. Let $G$ be a 3-edge-connected graph. If $I(G)>1$, then $G$ is a $P_{\geq 2}$-factor uniform graph.

Proof. For every $e=u v \in E(G)$, we write $G^{\prime}=G-e$. Since $G$ is 3-edge-connected, $G^{\prime}$ is connected. It suffices to justify that $G^{\prime}$ is a $P_{\geq 2}$-factor covered graph. Suppose, to the contrary, that $G^{\prime}$ is not $P_{\geq 2}$-factor covered. Then it follows from Lemma 1.1 that

$$
i\left(G^{\prime}-X\right) \geq 2|X|-\varepsilon_{1}(X)+1 .
$$

for some subset $X$ of $V\left(G^{\prime}\right)$.

If $X=\emptyset$, then by $(2.1)$ and $\varepsilon_{1}(X)=0$, we derive that $i\left(G^{\prime}\right) \geq 1$, contradicting that $G^{\prime}$ is connected. If $|X|=1$, then from $(2.1)$ and $\varepsilon_{1}(X) \leq 1$, we get that $i\left(G^{\prime}-X\right) \geq 2|X|=2$. Let $Q$ be the isolated vertex set of $G^{\prime}-X$. For $u \in Q$, we admit $d_{G^{\prime}-X}(u)=0$ and $d_{G}(u) \leq d_{G^{\prime}}(u)+1 \leq d_{G^{\prime}-X}(u)+|X|+1=d_{G^{\prime}-X}(u)+2=2$, which implies that $G$ is at most 2 -edge-connected. This contradicts that $G$ is 3 -edge-connected. If $|X|=2$, then we derive that

$$
i\left(G^{\prime}-X\right) \geq 2|X|-\varepsilon_{1}(X)+1 \geq 2|X|-1=3
$$

by $(2.1)$ and $\varepsilon_{1}(X) \leq 2$. Note that $i\left(G^{\prime}-X\right) \leq i(G-X)+2$. Thus, using (2.2) we obtain

$$
i(G-X) \geq i\left(G^{\prime}-X\right)-2 \geq 1 .
$$

Hence, $G-X$ has at least one isolated vertex, say for $w$, which implies that $w$ is only adjacent to some vertices in $X$. Note that $|X|=2$. Therefore, $G$ is at most 2-edge-connected. This contradicts that $G$ is 3 -edge-connected.

In what follows, we consider that $|X| \geq 3$. In light of $(2.1)$ and $\varepsilon_{1}(X) \leq 2$, we deduce

$$
i\left(G^{\prime}-X\right) \geq 2|X|-\varepsilon_{1}(X)+1 \geq 2|X|-1 \geq 6-1=5 .
$$

Combining this with $i\left(G^{\prime}-X\right) \leq i(G-X)+2$, we derive

$$
i(G-X) \geq i\left(G^{\prime}-X\right)-2 \geq 5-2=3 .
$$

From (2.1), (2.3), $\varepsilon_{1}(X) \leq 2$ and the definition of $I(G)$, we derive that

$$
I(G) \leq \frac{|X|}{i(G-X)} \leq \frac{|X|}{i\left(G^{\prime}-X\right)-2} \leq \frac{|X|}{2|X|-\varepsilon_{1}(X)-1} \leq \frac{|X|}{2|X|-3} .
$$

Let $f(|X|)=\frac{|X|}{2|X|-3}$. Then $\frac{\mathrm{d} f(|X|)}{\mathrm{d}|X|}=\frac{2|X|-3-2|X|}{(2|X|-3)^{2}}=-\frac{3}{(2|X|-3)^{2}}<0$, which implies that $f(|X|)$ attains its maximum value at $|X|=3$. Therefore, we derive by (2.4)

$$
I(G) \leq \frac{|X|}{2|X|-3} \leq \frac{3}{6-3}=1,
$$

which contradicts that $I(G)>1$. Theorem 2.1 is determined. 

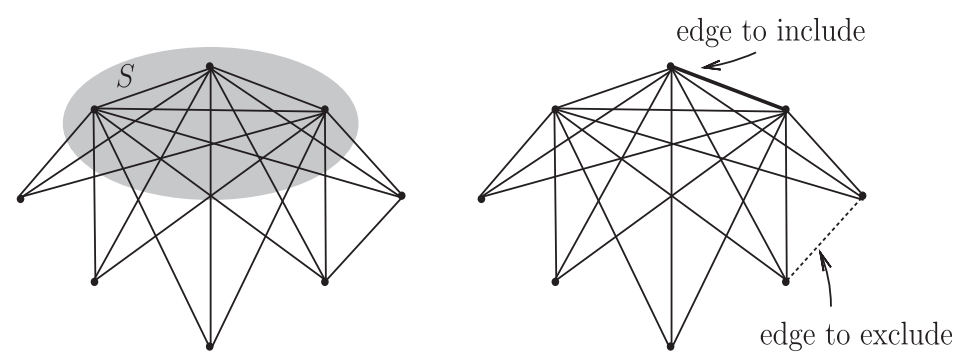

$$
I(G)=\frac{|S|}{i(G-S)}=1,
$$

$G$ is 3-edge-connected.

Figure 1. $G=K_{3} \vee\left(\left(3 K_{1}\right) \cup K_{2}\right)$.

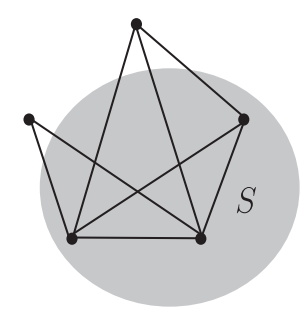

$G$ is 2-edge-connected,

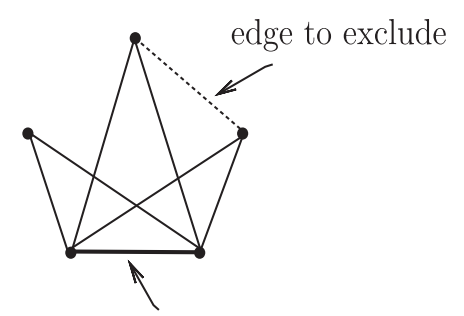

edge to include

$$
I(G)=\frac{|S|}{i(G-S)}=\frac{3}{2}>1 .
$$

Figure 2. $G=K_{2} \vee\left(K_{1} \cup K_{2}\right)$.

Remark 2.2. The condition that the isolated toughness $I(G)>1$ in Theorem 2.1 cannot be weakened to $I(G) \geq 1$. In order to explain this, let $G=K_{3} \vee\left(\left(3 K_{1}\right) \cup K_{2}\right)$ (see Fig. 1), where $\vee$ means "join". Trivially, $I(G)=1$ and $G$ is 3-edge-connected. Write $G^{\prime}=G-e$ for $e \in E\left(K_{2}\right)$. Let $X=V\left(K_{3}\right)$, and so $\varepsilon_{1}(X)=2$. Thus, we obtain

$$
i\left(G^{\prime}-X\right)=5>4=6-2=2|X|-\varepsilon_{1}(X) .
$$

By applying Lemma 1.1, $G^{\prime}$ is not $P_{\geq 2}$-factor covered, and so, $G$ is not a $P_{\geq 2}$-factor uniform graph.

Remark 2.3. The condition that $G$ is 3-edge-connected in Theorem 2.1 cannot be replaced by $G$ being 2 -edgeconnected. Let $G=K_{2} \vee\left(K_{1} \cup H\right)$ (see Fig. 2), where $H=K_{2}$. Clearly, $G$ is 2-edge-connected and $I(G)=\frac{3}{2}>1$. Set $G^{\prime}=G-e$ for $e \in E(H)$. Let $X=V\left(K_{2}\right)$, and so $\varepsilon_{1}(X)=2$. Hence, we derive

$$
i\left(G^{\prime}-X\right)=3>2=4-2=2|X|-\varepsilon_{1}(X) .
$$

From Lemma 1.1, $G^{\prime}$ is not $P_{\geq 2}$-factor covered, and so, $G$ is not a $P_{\geq 2}$-factor uniform graph.

\section{ISOLATED TOUGHNESS AND $P_{\geq 3}$-FACTOR UNIFORM GRAPHS}

Theorem 3.1. Let $G$ be a 3-edge-connected graph. Then $G$ is a $P_{\geq 3}$-factor uniform graph if its isolated toughness $I(G)>2$. 
Proof. For any $e=u v \in E(G)$, we write $G^{\prime}=G-e$. In order to testify Theorem 3.1, we need only to determine that $G^{\prime}$ is a $P_{\geq 3}$-factor covered graph. Suppose, to the contrary, that $G^{\prime}$ is not a $P_{\geq 3}$-factor covered graph. Then by Lemma 1.2 we have that

$$
\operatorname{sun}\left(G^{\prime}-X\right) \geq 2|X|-\varepsilon_{2}(X)+1
$$

for some subset $X$ of $V\left(G^{\prime}\right)$.

In what follows, the proof splits into two cases according to the value of $|X|$.

Case 1. $|X|=0$.

Apparently, $\varepsilon_{2}(X)=0$. In terms of (3.1), we derive

$$
\operatorname{sun}\left(G^{\prime}\right) \geq 1
$$

Note that $G$ is 3-edge-connected and $G^{\prime}=G-e$. Hence, $G^{\prime}$ is connected and $\operatorname{sun}\left(G^{\prime}\right) \leq \omega\left(G^{\prime}\right)=1$. Combining this with (3.2), we deduce

$$
\operatorname{sun}\left(G^{\prime}\right)=\omega\left(G^{\prime}\right)=1 .
$$

Since $G$ is 3-edge-connected, we get that $|V(G)|=\left|V\left(G^{\prime}\right)\right| \geq 4$. Combining this with the definitions of sun and big sun, $G^{\prime}$ is a big sun. Then $\left|V\left(G^{\prime}\right)\right| \geq 6$. Let $H$ be a factor-critical subgraph of $G^{\prime}$. Thus, we get that

$$
i\left(G^{\prime}-V(H)\right)=|V(H)|=\frac{\left|V\left(G^{\prime}\right)\right|}{2} \geq 3 .
$$

Subcase 1.1. $u \in V(H)$ or $v \in V(H)$.

Note that $e=u v \in E(G)$ and $G^{\prime}=G-e$. Then $i(G-V(H))=i\left(G^{\prime}-V(H)\right)$. Using (3.3), we have that $i(G-V(H))=|V(H)| \geq 3$. Thus, we deduce that

$$
I(G) \leq \frac{|V(H)|}{i(G-V(H))}=\frac{|V(H)|}{|V(H)|}=1
$$

contradicting that $I(G)>2$.

Subcase 1.2. $u \in V\left(G^{\prime}\right) \backslash V(H)$ and $v \in V\left(G^{\prime}\right) \backslash V(H)$.

Since $G^{\prime}$ is a big sun, there exists $w \in V(H)$ such that $v w \in E(G)$. Thus, we derive that $i(G-((V(H) \backslash$ $\{w\}) \cup\{v\}))=|V(G)|-|V(H)|=2|V(H)|-|V(H)|=|V(H)| \geq 3$. Hence, we deduce that

$$
I(G) \leq \frac{|(V(H) \backslash\{w\}) \cup\{v\}|}{i(G-((V(H) \backslash\{w\}) \cup\{v\}))}=\frac{|V(H)|}{|V(H)|}=1,
$$

it contradicts with $I(G)>2$.

Case 2. $|X| \geq 1$.

Claim 3.2. $\operatorname{sun}\left(G^{\prime}-X\right) \geq \max \{2,2|X|-1\}$.

Proof. If $|X|=1$, then it follows from (3.1) and $\varepsilon_{2}(X) \leq 1$ that

$$
\operatorname{sun}\left(G^{\prime}-X\right) \geq 2|X|-\varepsilon_{2}(X)+1 \geq 2|X|=2 .
$$

If $|X| \geq 2$, then by $(3.1)$ and $\varepsilon_{2}(X) \leq 2$ we have that

$$
\operatorname{sun}\left(G^{\prime}-X\right) \geq 2|X|-\varepsilon_{2}(X)+1 \geq 2|X|-1 .
$$

Thus, we derive that

$$
\operatorname{sun}\left(G^{\prime}-X\right) \geq \max \{2,2|X|-1\}
$$

for $|X| \geq 1$. This finishes the proof of Claim 3.2. 
Assume that $G^{\prime}-X$ has $a$ isolated vertices, $b K_{2}$ 's and $c$ big sun components $D_{1}, D_{2}, \cdots, D_{c}$, where $\left|V\left(D_{i}\right)\right| \geq 6$ for $i=1,2, \cdots, c$. Applying Claim 3.2, we deduce

$$
\operatorname{sun}\left(G^{\prime}-X\right)=a+b+c \geq \max \{2,2|X|-1\} \geq 2 .
$$

Let $W_{i}$ be the factor-critical subgraph of $D_{i}$ for $1 \leq i \leq c$, and setting $M_{i}=V\left(W_{i}\right)$ and $M=\bigcup_{i=1}^{c} M_{i}$. In terms of the definition of big sun, $i\left(D_{i}-M_{i}\right)=\left|M_{i}\right|=\frac{\left|V\left(D_{i}\right)\right|}{2}$. We select one vertex from every $K_{2}$ component of $G^{\prime}-X$, and denote the set of such vertices by $N$.

Subcase 2.1. $u \in V\left(a K_{1}\right)$ or $v \in V\left(a K_{1}\right)$.

Without loss of generality, we may assume that $u \in V\left(a K_{1}\right)$.

Subcase 2.1.1. $v \in V\left(a K_{1}\right)$.

Claim 3.3. $|X| \geq 2$.

Proof. Let $|X|=1$. Since $u$ and $v$ are two isolated vertices of $G^{\prime}-X$, we have $u x \notin E(G)$ and $v x \notin E(G)$ for any $x \in V(G) \backslash(X \cup\{u, v\})$. Thus, we derive $d_{G}(v) \leq|X|+1=2$, which implies that $G$ is at most 2-edge-connected, which contradicts that $G$ is 3 -edge-connected. Claim 3.3 is justified.

Note that $a \geq 2$ by $u \in V\left(a K_{1}\right)$ and $v \in V\left(a K_{1}\right)$. Since $G^{\prime}-X$ has $a$ isolated vertices, $b K_{2}$ 's and $c$ big sun components $D_{1}, D_{2}, \cdots, D_{c}, G-X$ has $(a-2)$ isolated vertices, $(b+1) K_{2}$ 's and $c$ big sun components $D_{1}, D_{2}, \cdots, D_{c}$. Thus, we get

$$
i(G-(X \cup M \cup N \cup\{u\}))=(a-2)+(b+1)+\sum_{i=1}^{c} \frac{\left|V\left(D_{i}\right)\right|}{2} \geq a+b+3 c-1 \geq a+b+c-1 .
$$

Using (3.4) and Claim 3.3, we obtain

$$
\operatorname{sun}\left(G^{\prime}-X\right)=a+b+c \geq 2|X|-1 \geq 3
$$

It follows from (3.5) and (3.6) that

$$
i(G-(X \cup M \cup N \cup\{u\}))=a+b-1+\sum_{i=1}^{c} \frac{\left|V\left(D_{i}\right)\right|}{2} \geq a+b+c-1 \geq 2 .
$$

In view of (3.7), the definition of $I(G)$ and $I(G)>2$, we deduce

$$
2<I(G) \leq \frac{|X \cup M \cup N \cup\{u\}|}{i(G-(X \cup M \cup N \cup\{u\}))}=\frac{|X|+|M|+|N|+1}{a+b-1+\sum_{i=1}^{c} \frac{\left|V\left(D_{i}\right)\right|}{2}}=\frac{|X|+\sum_{i=1}^{c} \frac{\left|V\left(D_{i}\right)\right|}{2}+b+1}{a+b-1+\sum_{i=1}^{c} \frac{\left|V\left(D_{i}\right)\right|}{2}},
$$

which implies

$$
|X|>2 a+b+\sum_{i=1}^{c} \frac{\left|V\left(D_{i}\right)\right|}{2}-3
$$

By applying (3.6), (3.8), $a \geq 2, c \geq 0$ and $\left|V\left(D_{i}\right)\right| \geq 6$, we acquire

$$
\frac{a+b+c+1}{2} \geq|X|>2 a+b+\sum_{i=1}^{c} \frac{\left|V\left(D_{i}\right)\right|}{2}-3 \geq 2 a+b+3 c-3 \geq a+b+c-1,
$$

that is,

$$
a+b+c<3
$$

contradicting (3.6). 
Subcase 2.1.2. $v \in V\left(b K_{2}\right)$.

There exists $w \in V\left(b K_{2}\right)$ with $v w \in E(G)$. In view of the definition of $N$, we know that one vertex in $\{v, w\}$ belongs to $N$. Let $Y \subseteq V\left(b K_{2}\right)$. Set $Y=N$ if $v \in N$, and $Y=(N \backslash\{w\}) \cup\{v\}$ if $v \notin N$. Then, it follows that

$$
i(G-(X \cup M \cup Y))=a+b+\sum_{i=1}^{c} \frac{\left|V\left(D_{i}\right)\right|}{2} \geq a+b+c \geq 2
$$

by (3.4). Combining this with the definition of $I(G)$ and $I(G)>2$, we derive

$$
2<I(G) \leq \frac{|X \cup M \cup Y|}{i(G-(X \cup M \cup Y))}=\frac{|X|+b+\sum_{i=1}^{c} \frac{\left|V\left(D_{i}\right)\right|}{2}}{a+b+\sum_{i=1}^{c} \frac{\left|V\left(D_{i}\right)\right|}{2}},
$$

and so,

$$
|X|>2 a+b+\sum_{i=1}^{c} \frac{\left|V\left(D_{i}\right)\right|}{2} \geq 2 a+b+3 c
$$

by $\left|V\left(D_{i}\right)\right| \geq 6$. Note that $a \geq 1$ and $c \geq 0$. In view of (3.4) and (3.9), we get that

$$
|X|>2 a+b+3 c \geq a+b+c+1 \geq(2|X|-1)+1=2|X|,
$$

this contradicts that $|X| \geq 1$.

Subcase 2.1.3. $v \in V\left(D_{1} \cup D_{2} \cup \cdots \cup D_{c}\right)$.

Without loss of generality, let $v \in V\left(D_{1}\right)$. Then there exists $w \in V\left(D_{1}\right)$ such that $v w \in E(G)$ and there is one vertex with degree 1 in $\{v, w\}$, in $D_{1}$. Thus, we derive

$$
i(G-(X \cup(M \backslash\{w\}) \cup\{v\} \cup N))=a+b+\sum_{i=1}^{c} \frac{\left|V\left(D_{i}\right)\right|}{2} \geq a+b+c \geq 2
$$

by (3.4), $c \geq 0$ and $\left|V\left(D_{i}\right)\right| \geq 6$. In terms of (3.10) and $I(G)>2$, we get

$$
2<I(G) \leq \frac{|X \cup(M \backslash\{w\}) \cup\{v\} \cup N|}{i(G-(X \cup(M \backslash\{w\}) \cup\{v\} \cup N))}=\frac{|X|+|M|+|N|}{a+b+\sum_{i=1}^{c} \frac{\left|V\left(D_{i}\right)\right|}{2}}=\frac{|X|+\sum_{i=1}^{c} \frac{\left|V\left(D_{i}\right)\right|}{2}+b}{a+b+\sum_{i=1}^{c} \frac{\left|V\left(D_{i}\right)\right|}{2}},
$$

and so,

$$
2 a+b+\sum_{i=1}^{c} \frac{\left|V\left(D_{i}\right)\right|}{2}-|X|<0
$$

It follows from (3.4), (3.11), $a \geq 1, c \geq 0,\left|V\left(D_{i}\right)\right| \geq 6$ and $|X| \geq 1$ that

$$
0>2 a+b+\sum_{i=1}^{c} \frac{\left|V\left(D_{i}\right)\right|}{2}-|X| \geq a+b+c-|X|+1 \geq 2|X|-1-|X|+1=|X| \geq 1,
$$

this is a contradiction. 
Subcase 2.1.4. $v \in V(G) \backslash\left(V\left(a K_{1}\right) \cup V\left(b K_{2}\right) \cup V\left(D_{1} \cup D_{2} \cup \cdots \cup D_{c}\right)\right)$.

In this subcase, we easily deduce that

$$
i(G-(X \cup M \cup N \cup\{v\})) \geq a+b+\sum_{i=1}^{c} \frac{\left|V\left(D_{i}\right)\right|}{2} \geq a+b+c \geq 2
$$

according to (3.4), $c \geq 0$ and $\left|V\left(D_{i}\right)\right| \geq 6$.

From (3.12) and $I(G)>2$, we acquire that

$$
2<I(G) \leq \frac{|X \cup M \cup N \cup\{v\}|}{i(G-(X \cup M \cup N \cup\{v\}))} \leq \frac{|X|+|M|+|N|+1}{a+b+\sum_{i=1}^{c} \frac{\left|V\left(D_{i}\right)\right|}{2}}=\frac{|X|+\sum_{i=1}^{c} \frac{\left|V\left(D_{i}\right)\right|}{2}+b+1}{a+b+\sum_{i=1}^{c} \frac{\left|V\left(D_{i}\right)\right|}{2}},
$$

and so,

$$
|X|>2 a+b+\sum_{i=1}^{c} \frac{\left|V\left(D_{i}\right)\right|}{2}-1
$$

In light of (3.4), (3.13), $a \geq 1, c \geq 0$ and $\left|V\left(D_{i}\right)\right| \geq 6$, we have that

$$
\begin{aligned}
a+b+c & \geq 2|X|-1>2\left(2 a+b+\sum_{i=1}^{c} \frac{\left|V\left(D_{i}\right)\right|}{2}-1\right)-1 \\
& =4 a+2 b+\sum_{i=1}^{c}\left|V\left(D_{i}\right)\right|-3 \\
& \geq 4 a+2 b+6 c-3 \geq 2(a+b+c)-1 \\
& \geq a+b+c+1,
\end{aligned}
$$

this is a contradiction.

Subcase 2.2. $u \notin V\left(a K_{1}\right)$ and $v \notin V\left(a K_{1}\right)$.

Claim 3.4. $I(G) \leq \frac{|X|+b+\sum_{i=1}^{c} \frac{\left|V\left(D_{i}\right)\right|}{2}}{a+b+\sum_{i=1}^{c} \frac{\left|V\left(D_{i}\right)\right|}{2}}$.

Proof. We discuss the following three cases.

Subcase 2.2.1. $u \in V\left(b K_{2}\right)$ or $v \in V\left(b K_{2}\right)$.

Without loss of generality, let $u \in V\left(b K_{2}\right)$. Then there exists $w \in V\left(b K_{2}\right)$ such that $u w \in E\left(b K_{2}\right)$.

Let $Y \subseteq V\left(b K_{2}\right)$. Setting $Y=N$ if $u \in N$, and $Y=(N \backslash\{w\}) \cup\{u\}$ if $u \notin N$. Thus, we obtain

$$
i(G-(X \cup M \cup Y))=a+b+\sum_{i=1}^{c} \frac{\left|V\left(D_{i}\right)\right|}{2} \geq a+b+c \geq 2
$$

in terms of (3.4), $c \geq 0$ and $\left|V\left(D_{i}\right)\right| \geq 6$. Combining this with the definition of $I(G)$, we get that

$$
I(G) \leq \frac{|X \cup M \cup Y|}{i(G-(X \cup M \cup Y))}=\frac{|X|+|M|+|Y|}{a+b+\sum_{i=1}^{c} \frac{\left|V\left(D_{i}\right)\right|}{2}}=\frac{|X|+b+\sum_{i=1}^{c} \frac{\left|V\left(D_{i}\right)\right|}{2}}{a+b+\sum_{i=1}^{c} \frac{\left|V\left(D_{i}\right)\right|}{2}}
$$


Subcase 2.2.2. $u \in V\left(D_{1} \cup D_{2} \cup \cdots \cup D_{c}\right)$ or $v \in V\left(D_{1} \cup D_{2} \cup \cdots \cup D_{c}\right)$.

Without loss of generality, let $u \in V\left(D_{1}\right)$. Then there exists $w \in V\left(D_{1}\right)$ such that $u w \in E(G)$ and there is one vertex with degree 1 in $\{u, w\}$, in $D_{1}$. Hence, we get

$$
i(G-(X \cup(M \backslash\{w\}) \cup\{u\} \cup N))=a+b+\sum_{i=1}^{c} \frac{\left|V\left(D_{i}\right)\right|}{2} \geq a+b+c \geq 2
$$

in light of (3.4), $c \geq 0$ and $\left|V\left(D_{i}\right)\right| \geq 6$. Thus, we acquire

$$
I(G) \leq \frac{|X \cup(M \backslash\{w\}) \cup\{u\} \cup N|}{i(G-(X \cup(M \backslash\{w\}) \cup\{u\} \cup N))}=\frac{|X|+|M|+|N|}{a+b+\sum_{i=1}^{c} \frac{\left|V\left(D_{i}\right)\right|}{2}}=\frac{|X|+b+\sum_{i=1}^{c} \frac{\left|V\left(D_{i}\right)\right|}{2}}{a+b+\sum_{i=1}^{c} \frac{\left|V\left(D_{i}\right)\right|}{2}} .
$$

Subcase 2.2.3. $u, v \in V(G) \backslash\left(V\left(a K_{1}\right) \cup V\left(b K_{2}\right) \cup V\left(D_{1} \cup D_{2} \cup \cdots \cup D_{c}\right)\right)$.

In this subcase, we have $i(G-(X \cup M \cup N))=i\left(G^{\prime}-(X \cup M \cup N)\right)=a+b+\sum_{i=1}^{c} \frac{\left|V\left(D_{i}\right)\right|}{2}$. Combining this with (3.4), $c \geq 0$ and $\left|V\left(D_{i}\right)\right| \geq 6$, we admit

$$
i(G-(X \cup M \cup N))=a+b+\sum_{i=1}^{c} \frac{\left|V\left(D_{i}\right)\right|}{2} \geq a+b+c \geq 2 .
$$

It follows from (3.15) that

$$
I(G) \leq \frac{|X \cup M \cup N|}{i(G-(X \cup M \cup N))}=\frac{|X|+|M|+|N|}{a+b+\sum_{i=1}^{c} \frac{\left|V\left(D_{i}\right)\right|}{2}}=\frac{|X|+b+\sum_{i=1}^{c} \frac{\left|V\left(D_{i}\right)\right|}{2}}{a+b+\sum_{i=1}^{c} \frac{\left|V\left(D_{i}\right)\right|}{2}} .
$$

Claim 3.4 is determined.

Using $I(G)>2$ and Claim 3.4, we derive

$$
2<I(G) \leq \frac{|X|+b+\sum_{i=1}^{c} \frac{\left|V\left(D_{i}\right)\right|}{2}}{a+b+\sum_{i=1}^{c} \frac{\left|V\left(D_{i}\right)\right|}{2}}
$$

that is,

$$
|X|>2 a+b+\sum_{i=1}^{c} \frac{\left|V\left(D_{i}\right)\right|}{2} .
$$

It follows from (3.4), (3.16), $a \geq 0, c \geq 0,\left|V\left(D_{i}\right)\right| \geq 6$ and $|X| \geq 1$ that

$$
|X|>2 a+b+\sum_{i=1}^{c} \frac{\left|V\left(D_{i}\right)\right|}{2} \geq a+b+c \geq 2|X|-1 \geq|X|,
$$

it is a contradiction. Theorem 3.1 is demonstrated. 


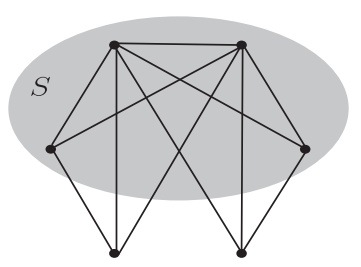

$I(G)=\frac{|S|}{i(G-S)}=2$,

$G$ is 3-edge-connectd.

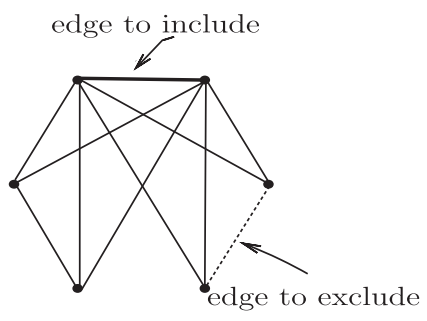

Figure $3 . G=K_{2} \vee\left(2 K_{2}\right)$.
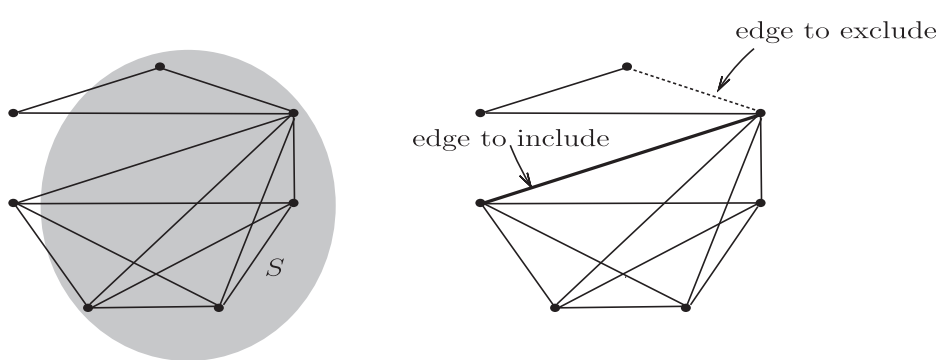

$G$ is 2-edge-connected,

$I(G)=\frac{|S|}{i(G-S)}=\frac{5}{2}>2$.

FigURE 4. $G=K_{1} \vee\left(K_{2} \cup K_{4}\right)$.

Remark 3.5. The condition that isolated toughness $I(G)>2$ in Theorem 3.1 cannot be weakened to $I(G) \geq 2$. In order to see this, let $G=K_{2} \vee H$ (see Fig. 3), where $H=2 K_{2}$. Then $I(G)=2$ and $G$ is 3-edge-connected. Put $G^{\prime}=G-e$ for $e \in E(H)$. Let $X=V\left(K_{2}\right)$, and so $\varepsilon_{2}(X)=2$. Thus, we derive

$$
\operatorname{sun}\left(G^{\prime}-X\right)=3>2=4-2=2|X|-\varepsilon_{2}(X) .
$$

By Lemma 1.2, $G^{\prime}$ is not $P_{\geq 3}$-factor covered, and so, $G$ is not a $P_{\geq 3}$-factor uniform graph.

Remark 3.6. The condition that $G$ is 3 -edge-connected in Theorem 3.1 cannot be replaced by $G$ being 2 edge-connected. In order to show this, let $G=K_{1} \vee\left(K_{2} \cup K_{4}\right)$ (see Fig. 4). Then $G$ is 2-edge-connected and $I(G)=\frac{5}{2}>2$. Setting $G^{\prime}=G-e$ for $e \in E\left(K_{2}\right)$. Let $X=V\left(K_{1}\right)$, and so $\varepsilon_{2}(X)=1$. Therefore, we have that

$$
\operatorname{sun}\left(G^{\prime}-X\right)=2>1=2-1=2|X|-\varepsilon_{2}(X) .
$$

By Lemma $1.2, G^{\prime}$ is not $P_{\geq 3}$-factor covered, and so, $G$ is not a $P_{\geq 3}$-factor uniform graph.

\section{Conclusion}

In this paper, we reveal the relationship between isolated toughness of the network and the existence of path factors in the network, and derive two results for the existence of path factors in networks which are summarized as follows:

- A 3-edge-connected graph $G$ is a $P_{\geq 2}$-factor uniform graph if its isolated toughness $I(G)>1$. 
- A 3-edge-connected graph $G$ is a $P_{\geq 3}$-factor uniform graph if its isolated toughness $I(G)>2$.

Furthermore, we explain that the conditions in our main results are sharp. Path factors in graphs or networks have attracted a great deal of attention due to their applications in network design, statistical mechanics, information transmission in networks, and so on. Therefore, there is theoretical and practical significance in investigating the problem of path factors in graphs or networks.

Further, we present the following open problems at the end of our paper.

Problem 1. What is the relationship between isolated toughness of the network and the existence of other types of path factors in the network?

Problem 2. What is the relationship between other parameters of the network and the existence of path factors in the network?

Acknowledgements. The authors would like to thank the anonymous reviewers for their kind comments and valuable suggestions. This work is supported by Six Talent Peaks Project in Jiangsu Province, China (Grant No. JY-022).

\section{REFERENCES}

[1] S. Abdullah and M. Aslam, New multicriteria group decision support systems for small hydropower plant locations selection based on intuitionistic cubic fuzzy aggregation information. Int. J. Intell. Syst. 35 (2020) 983-1020.

[2] J. Akiyama, D. Avis and H. Era, On a \{1,2\}-factor of a graph. TRU Math. 16 (1980) 97-102.

[3] F. Chiclana, F. Mata, L. Pérez and E. Herrera-Viedma, Type-1 OWA unbalanced fuzzy linguistic aggregation methodology: application to eurobonds credit risk evaluation. Int. J. Intell. Syst. 33 (2018) 1071-1088.

[4] W. Gao and W. Wang, New isolated toughness condition for fractional $(g, f, n)$-critical graphs. Colloquium Math. 147 (2017) $55-66$.

[5] W. Gao, L. Liang and Y. Chen, An isolated toughness condition for graphs to be fractional (k, m)-deleted graphs. Util. Math. 105 (2017) 303-316.

[6] W. Gao, W. Wang and Y. Chen, Tight bounds for the existence of path factors in network vulnerability parameter settings. Int. J. Intell. Syst. 36 (2021) 1133-1158.

[7] A. Kaneko, A necessary and sufficient condition for the existence of a path factor every component of which is a path of length at least two. J. Comb. Theory Ser. B 88 (2003) 195-218.

[8] M. Kano, G.Y. Katona and Z. Király, Packing paths of length at least two. Discrete Math. 283 (2004) $129-135$.

[9] M. Kano, C. Lee and K. Suzuki, Path and cycle factors of cubic bipartite graphs. Discuss. Math. Graph Theory 28 (2008) $551-556$.

[10] J. Li, H. Yan, Z. Liu, X. Chen, X. Huang and D. Wong, Location-sharing systems with enhanced privacy in mobile online social networks. IEEE Syst. J. 11 (2017) 439-448.

[11] J. Li, L. Sun, Q. Yan, Z. Li, W. Srisa-An and H. Ye, Significant permission identification for machine-learning-based android malware detection. IEEE Trans. Ind. Inf. 14 (2018) 3216-3225.

[12] Z. Sun and S. Zhou, A generalization of orthogonal factorizations in digraphs. Inf. Process. Lett. 132 (2018) $49-54$.

[13] H. Wang, Path factors of bipartite graphs. J. Graph Theory 18 (1994) 161-167.

[14] S. Wang and W. Zhang, Research on fractional critical covered graphs. Prob. Inf. Transm. 56 (2020) $270-277$.

[15] S. Wang and W. Zhang, On k-orthogonal factorizations in networks. RAIRO:OR 55 (2021) 969-977.

[16] J. Yang, Y. Ma and G. Liu, Fractional ( $g, f)$-factors in graphs. Appl. Math. - A J. Chin. Univ. Ser. A 16 (2001) 385-390.

[17] H. Zhang and S. Zhou, Characterizations for $P_{\geq 2}$-factor and $P_{\geq_{3}}$-factor covered graphs. Discrete Math. 309 (2009) $2067-2076$.

[18] S. Zhou, Some results about component factors in graphs. RAIRO:OR 53 (2019) 723-730.

[19] S. Zhou, Remarks on path factors in graphs. RAIRO:OR 54 (2020) 1827-1834.

[20] S. Zhou, Some results on path-factor critical avoidable graphs. Discuss. Math. Graph Theory (2020). DOI: 10.7151/dmgt.2364.

[21] S. Zhou, Binding numbers and restricted fractional $(g, f)$-factors in graphs. Discrete Appl. Math. (2020). DOI: 10.1016/j.dam.2020.10.017.

[22] S. Zhou and Z. Sun, Binding number conditions for $P_{\geq 2}$-factor and $P_{\geq 3}$-factor uniform graphs. Discrete Math. 343 (2020) 111715.

[23] S. Zhou and Z. Sun, A neighborhood condition for graphs to have restricted fractional ( $g$, f)-factors. Contrib. Discrete Math. 16 (2021) 138-149.

[24] S. Zhou, F. Yang and L. Xu, Two sufficient conditions for the existence of path factors in graphs. Sci. Iran. 26 (2019) $3510-3514$.

[25] S. Zhou, Y. Xu and Z. Sun, Degree conditions for fractional $(a, b, k)$-critical covered graphs. Inf. Process. Lett. 152 (2019) 105838 . 
[26] S. Zhou, Z. Sun and Q. Pan, A sufficient condition for the existence of restricted fractional ( $g$, $f$ )-factors in graphs. Prob. Inf. Transm. 56 (2020) 332-344.

[27] S. Zhou, T. Zhang and Z. Xu, Subgraphs with orthogonal factorizations in graphs. Discrete Appl. Math. 286 (2020) $29-34$.

[28] S. Zhou, Q. Bian and Z. Sun, Two sufficient conditions for component factors in graphs. Discuss. Math. Graph Theory (2021). DOI: $10.7151 / \mathrm{dmgt.} 2401$.

[29] S. Zhou, H. Liu and Y. Xu, A note on fractional ID-[a,b]-factor-critical covered graphs. Discrete Appl. Math. (2021). DOI: 10.1016/j.dam.2021.03.004. 